\title{
Correction to: Wormlike micelles formed by ultra-long-chain nonionic surfactant
}

\author{
Jiuxia Wang ${ }^{1} \cdot$ Yongmin Zhang $^{1,3} \cdot$ Zonglin Chu $^{1} \cdot$ Yujun Feng ${ }^{1,2}$
}

Published online: 7 August 2021

○) Springer-Verlag GmbH Germany, part of Springer Nature 2021

\section{Colloid and polymer science https://doi.org/ 10.1007/s00396-021-04848-z}

In this article, "Yujun Feng" should have been denoted as a corresponding author.

The original article has been corrected.

Publisher's Note Springer Nature remains neutral with regard to jurisdictional claims in published maps and institutional affiliations.

The original article can be found online at https://doi.org/10.1007/ s00396-021-04848-z.

Yongmin Zhang

zhangym@jiangnan.edu.cn

$\triangle$ Yujun Feng

yjfeng@scu.edu.cn

1 Chengdu Institute of Organic Chemistry, Chinese Academy of Sciences, Chengdu 610041, People's Republic of China

2 State Key Laboratory of Polymer Materials Engineering, Polymer Research Institute, Chengdu 610065,

People's Republic of China

3 The Key Laboratory of Synthetic and Biological Colloids, Ministry of Education, School of Chemical \& Materials Engineering, Jiangnan University, Wuxi 214122,

People's Republic of China 